\title{
Comportamiento volumétrico de la DL-VAlina en soluciones ACUOSAS DE NITRATO DE SODIO A DIFERENTES TEMPERATURAS
}

\author{
Manuel Páez-Meza'* , Jorge Ramos-Montiel ${ }^{1}$ \\ y Nicolás De La Espriella-Vélez ${ }^{2}$ \\ ${ }^{1}$ Programa de Química, Facultad de Ciencias Básicas. ${ }^{2}$ Programa de \\ Física, Grupo GAMASCO, Facultad de Ciencias Básicas, Universidad de \\ Córdoba, C.P. 230002, Montería, Colombia. E-mail: *mspaezm@gmail.com
}

\begin{abstract}
Resumen
Se determinaron las densidades de la DL-valina (Ácido 2-amino-3-metilbutanoico) en soluciones acuosas de nitrato de sodio en el intervalo de temperaturas desde $283.15 \mathrm{~K}$ hasta $318.15 \mathrm{~K}$ usando un densímetro de tubo vibratorio Anton Paar DMA 5000. Se calcularon: los volúmenes molares aparentes, los volúmenes molares aparentes a dilución infinita, la segunda derivada de los volúmenes molares parciales a dilución infinita con respecto a la temperatura, así como los volúmenes molares parciales de transferencia y los números de hidratación. Los resultados obtenidos se discutieron en términos de las interacciones predominantes en solución, encontrándose que la DL-valina tiene un efecto disruptor de la estructura del solvente y que a dilución infinita predominan las interacciones soluto-solvente entre el grupo isopropil del aminoácido y los iones sodio y nitrato.
\end{abstract}

Palabras Clave: densidad, DL-valina, soluciones acuosas, volumen molar aparente, volumen molarparcial de transferencia.

\section{Volumetric behavior of the DL-valine in aqueous solutions of sodium nitrate at different temperatures}

\begin{abstract}
Densities of DL-valine (2-amino-3-methylbutanoic acid) in aqueous solutions of sodium nitrate were determined at temperatures ranging from 283.15 to $318.15 \mathrm{~K}$ using an Anton Paar DMA 5000 vibrating tube densitometer. The apparent molar volume, infinite dilution apparent molar volume, second derivative of the infinite dilution partial molar volume with respect to temperature, partial molar volume of transfer at infinite dilution and the number of hydration were calculated. The results obtained were discussed in terms of the dominant interactions in solution, it was found that the DL-valine has a disruptor effect in the structure of the solvent and that at infinite dilution solute-solvent interactions are dominant between isopropyl group the amino acid and sodium and nitrate ions.
\end{abstract}

Key Words: density, DL-valine, aqueous solution, apparent molar volume, partial molar volume of transfer. 


\section{INTRODUCCIÓN}

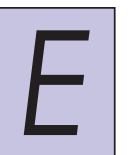

s bien conocido que el comportamiento y las propiedades moleculares de las proteínas en las mezclas acuosas es gobernado por muchos factores, como: estructura química, propiedades del disolvente, $\mathrm{pH}$, electrolitos presentes en solución, entre otras ${ }^{1}$. La fuerte interacción entre electrolitos y proteínas es la causa de la desviación del comportamiento ideal. Por tanto, este hecho es quizás una de las razones por la que debe estudiarse la influencia de los electrolitos sobre el comportamiento fisicoquímico de las proteínas.

No obstante, ya que este estudio resulta inviable por no decir imposible, debido a la compleja organización estructural de estas macromoléculas biológicas, se recurre al estudio de las interacciones de los bloques de construcción de las proteínas (aminoácidos) en soluciones acuosas de electrolitos, con el fin de entender los finos detalles asociados al comportamiento volumétrico ${ }^{2}$. Por esta razón, es evidente que un estudio directo de las proteínas resulta difícil ${ }^{3}$ y debido a ello en los últimos años se han usado diversos aminoácidos y péptidos de bajo peso molecular como compuestos modelos en soluciones salinas, con el fin de simular estos complejos ambientes biológicos ${ }^{4-7}$. De hecho, existen extensos estudios de propiedades volumétricas y termoquímicas sobre esta área de investigación ${ }^{8-11}$.

Además de los argumentos expuestos, otra razón no menos importante está asociada al hecho de que los aminoácidos y péptidos son las unidades estructurales fundamentales de las proteínas, péptidos, ciertos tipos de hormonas, antibióticos y muchos otros compuestos de relevanciabiológica. Generalmente, se reconoce que en ausencia de datos termodinámicos experimentales para estas macromoléculas, los aminoácidos y péptidos pueden servir como modelos útiles para la estimación de sus propiedades ${ }^{12-15}$.

En virtud a lo anterior y dada la trascendencia del tema, en este trabajo se estudian algunas propiedades volumétricas de soluciones de DL-valina en mezclas acuosas de nitrato de sodio a diferentes concentraciones y a las temperaturas de $283.15,288.15,293.15,298.15,303.15,308.15,313.15$ y $318.15 \mathrm{~K}$, con el fin de predecir el efecto que causa la adición de una sal sobre el comportamiento de los aminoácidos. Los resultados obtenidos se discuten en términos de las interacciones predominantes en solución.

\section{MAteriales y mÉtOdos Reactivos}

Se empleó DL-valina (Alfa Aesar) y nitrato de sodio (SigmaAldrich) ambos con una pureza del $99 \%$. Antes de su uso la DL-valina fue recristalizada dos veces en soluciones acuosas de etanol y secada al vacío a $348 \mathrm{~K}$ durante $6 \mathrm{~h}$, después se almacenó en un desecador sobre $\mathrm{P}_{2} \mathrm{O}_{5}$. El agua utilizada fue desgasificada y doblemente destilada en medio alcalino con $\mathrm{KMnO}_{4}$ para eliminar la materia orgánica ${ }^{16}$, obteniéndose con una conductividad inferior a $2 \mu \mathrm{S} \mathrm{cm} \mathrm{cm}^{-1}$.

\section{Preparación de las soluciones}

En este trabajo, se prepararon cinco solventes acuosos de nitrato de sodio a concentraciones de $(0.0000,0.0106,0.0413,0.1009$, 0.4013 y 0.9014$) \mathrm{mol} \mathrm{kg}^{-1}$ que fueron mezclados con cantidades específicas de DL-valina hasta obtener concentraciones en el intervalo de 0.0000 mol kg-1 hasta $0.4000 \mathrm{~mol} \mathrm{~kg}^{-1}$. Las incertidumbres relativas (expresadas en porcentaje) para las concentraciones molales se encontraron en el intervalo de $0.02 \%$ a $0.28 \%$, usando la ecuación (1):

$(\Delta m) \frac{100}{m}=\left(\frac{1000}{M}\left[\frac{W_{2}}{W_{1}^{2}} \Delta W+\frac{\Delta W}{W_{1}}\right]+\frac{1000 W_{2}}{W_{1}}\left[\frac{\Delta M}{M^{2}}\right]\right) \frac{100}{m}$

Donde $M$ es la masa molar de la DL-valina, $W_{2}$ y $W_{1}$ es la masa de soluto y solvente respectivamente, $\Delta M$ es la incertidumbre asociada a la masa molar de la DL-valina y $\Delta W$ es la incertidumbre de la balanza analítica utilizada marca Precisa $\left( \pm 1 \times 10^{-5} \mathrm{~g}\right)$.

\section{Determinación de la densidad}

Las densidades de las soluciones fueron determinadas en un densímetro de tubo vibratorio Anton Paar DMA 5000, con una incertidumbre de $\pm 1 \times 10^{-5} \mathrm{~g} \mathrm{~cm}^{-3}$ y un control de temperatura de $\pm 0.001 \mathrm{~K}$ desde $283.15 \mathrm{~K}$ hasta $318.15 \mathrm{~K}$ a intervalos de $5 \mathrm{~K}$. Se verificó el buen funcionamiento del equipo, determinando la densidad del agua bidestilada a diferentes temperaturas, y los datos obtenidos resultaron en buen acuerdo con los reportados en literatura ${ }^{17-18}$ como se observa en la Tabla I.

\section{RESULTADOS Y DISCUSIÓN}

Las densidades obtenidas para la DL-valina en mezclas acuosas de $\mathrm{NaNO}_{3}$ aumentan conforme incrementan las concentraciones del aminoácido y del nitrato de sodio, pero disminuyen con el

\begin{tabular}{|c|c|c|c|c|c|c|c|c|c|c|}
\hline \multicolumn{2}{|c|}{$\mathrm{T} / \mathrm{K}$} & 283.15 & 288.15 & 293.15 & 298.15 & 303.15 & 308.15 & 313.15 & 318.15 \\
\hline \multirow{3}{*}{ Agua } & \multicolumn{7}{|c|}{$\rho / \mathrm{cm}^{-3}$} \\
\cline { 2 - 11 } & a & 0.99969 & 0.99909 & 0.99819 & 0.99704 & 0.99564 & 0.99402 & 0.99221 & 0.99020 \\
\cline { 2 - 11 } & ${ }^{\mathrm{b}}$ & 0.99973 & 0.99913 & 0.99823 & 0.99707 & 0.99568 & 0.99406 & 0.99224 & 0.99024 \\
\cline { 2 - 11 } & c & 0.9997 & 0.9991 & 0.9982 & 0.9970 & 0.9956 & 0.9940 & 0.9922 & 0.9902 \\
\hline
\end{tabular}

Tabla I. Densidades del agua bidestilada a diferentes temperaturas: a. Este trabajo, b. Ref. ${ }^{17}$ y c. Ref. ${ }^{18}$. 
incremento de la temperatura como se observa en las Figuras 1 y 2 respectivamente.

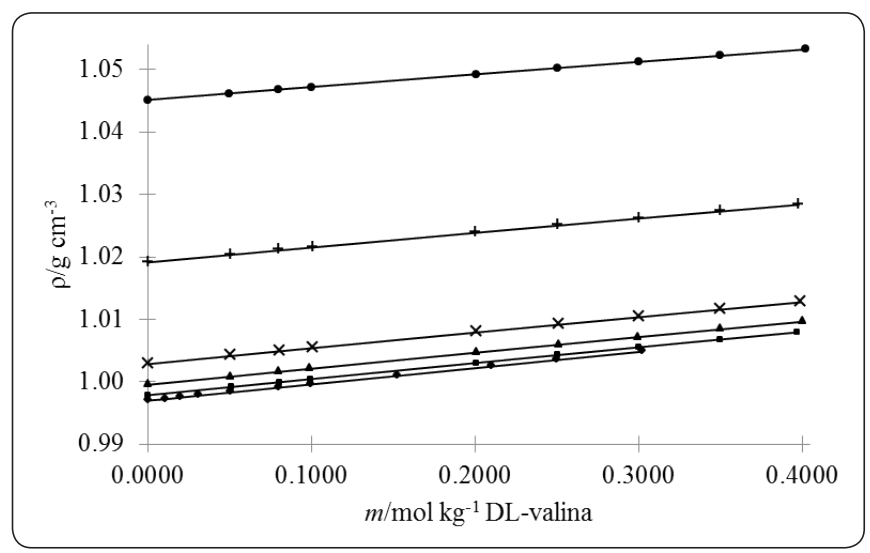

Figura 1. Densidades de la DL-valina en mezclas acuosas de $\mathrm{NaNO}_{3}(\square 0.0000, \times 0.0106, \triangle 0.0413, \triangleleft 0.1009,+0.4013, \bullet$ 0.9014 ) $\mathrm{mol} \mathrm{kg}^{-1}$ a temperatura de $298.15 \mathrm{~K}$.

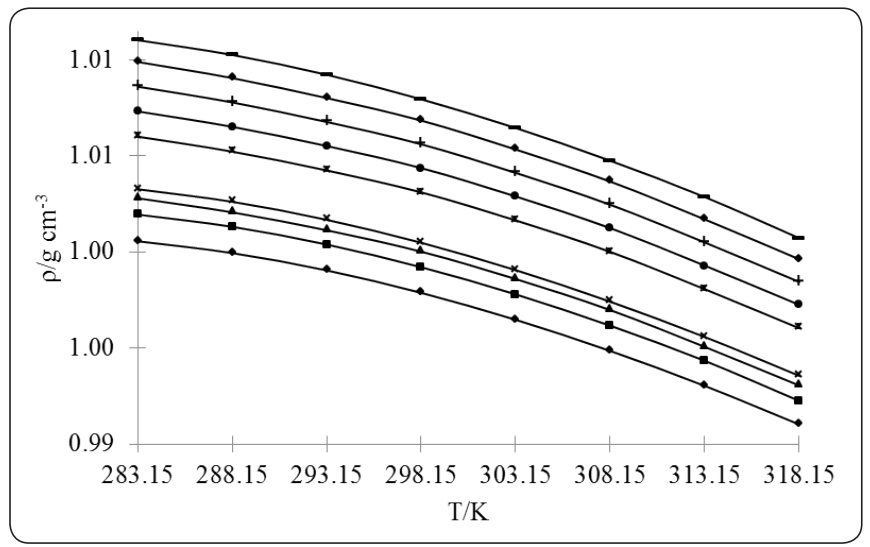

Figura 2. Densidades de la DL-valina en mezcla acuosa de $\mathrm{NaNO}_{3} 0.0106 \mathrm{~mol} \mathrm{~kg}^{-1}$ a diferentes temperaturas. (Todos los sistemas acuosos de $\mathrm{NaNO}_{3}$ estudiados presentan la misma tendencia en sus datos).

Los valores de los volúmenes molares aparentes $V_{\phi}$ para la DL-valina en estas mezclas acuosas de $\mathrm{NaNO}_{3}$, fueron calculados a partir de los datos de densidad utilizando la ecuación $(2)^{19}$.

$V_{\phi}=\frac{M}{\rho}-\frac{1000\left(\rho-\rho_{0}\right)}{m \rho \rho_{0}}$

Donde $M$ es la masa molar de la DL-valina, $m$ es la molalidad del aminoácido en las mezclas acuosas de $\mathrm{NaNO}_{3}, \rho$ y $\rho_{0}$ son la densidad de la solución (DL-valina en mezclas acuosas de
$\mathrm{NaNO}_{3}$ ) y la densidad del solvente (mezclas acuosas de $\mathrm{NaNO}_{3}$ ) respectivamente. Las incertidumbres relativas (expresadas en porcentaje) para los volúmenes molares se encontraron en el intervalo de $0.1 \%$ a $2.0 \%$, usando la ecuación (3):

$\left(\Delta V_{\phi}\right) \frac{100}{V_{\phi}}=\left(\frac{\Delta M}{\rho}+\frac{M}{\rho^{2}} \Delta \rho+\frac{1000 m\left(\rho^{2}+\rho_{0}^{2}\right.}{\left(m \rho \rho_{0}\right)^{2}} \Delta \rho+\frac{1000\left(\rho-\rho_{0}\right)}{m^{2} \rho \rho_{0}} \Delta m\right) \frac{100}{V_{\phi}}$

Donde $\Delta \rho$ es la incertidumbre del densímetro y $\Delta m$ es la incertidumbre asociada a la concentración de las soluciones. El término de mayor incertidumbre resulta ser el tercero dentro del paréntesis al lado derecho de la ecuación (3).

Los resultados para las densidades y los volúmenes molares aparentes obtenidos son reportados en la Tabla II. La variación de $V_{\phi}$ con respecto a la concentración molal se correlacionó adecuadamente por medio de la ecuación de Masson a las temperaturas de trabajo ${ }^{20}$, a fin de evaluar los volúmenes molares aparentes a dilución infinita $V_{\phi}^{0}$, utilizando la ecuación (4). Para ello se toma la región de mayor concentración donde la tendencia de $V_{\phi}$ en función de $m^{1 / 2}$ es lineal; este procedimiento para el cálculo de $V_{\phi}^{0}$ ha sido previamente utilizado por Frank y Smith $^{21}$, y es comúnmente aceptado debido a que la incertidumbre experimental de $V_{\phi}$ aumenta a medida que la concentración disminuye.

$V_{\phi}=V_{\phi}^{0}+S_{V} m^{1 / 2}$

Donde $S_{V}$ es la pendiente límite experimental ${ }^{22}$.

En la región de dilución infinita, el volumen molar aparente es igual al volumen molar parcial de la DL-valina $\left(V_{\phi}^{0}=\bar{V}_{2}^{0}\right)^{23}$, y los resultados obtenidos para $V_{\phi}^{0}$ se presentan en la Tabla III, observándose que ellos disminuyen con el incremento de la concentración del cosolvente $\mathrm{NaNO}_{3}$, lo cual puede ser interpretado como una contracción del volumen a dilución infinita debido al incremento de las interacciones solutocosolvente. Así mismo, los valores de $V_{\phi}^{0}$ aumentan con el aumento de la temperatura, lo que podría ser atribuido a un debilitamiento de las interacciones que resultan del incremento de la energía cinética de las partículas.

También se analizó el efecto de la temperatura sobre el comportamiento del volumen molar aparente a dilución infinita $V_{\phi}^{0}$ y éste se discute en términos de las interacciones hidrofóbicas e hidrofílicas y efecto del soluto sobre la estructura del solvente ${ }^{14}$. Para lo cual, los valores experimentales de $V_{\phi}^{0}$ fueron correlacionados con la temperatura usando la ecuación (5).

$V_{\phi}^{0}=A+B T+C T^{2}$

Donde $A, B$ y $C$ son parámetros ajustables y $T$ es la temperatura absoluta. En particular para estos sistemas los valores de $\partial^{2} V_{\phi}^{0}$ $/ \partial T^{2}$ resultaron positivos para la mezcla binaria DL-valina 
+ agua y negativos para la DL-valina + mezclas acuosas de $\mathrm{NaNO}_{3}$, como se observa en la Tabla IV. Lo cual indica, según Hepler que en el primer sistema la DL-valina tiene un efecto formador de la estructura del agua; mientras que en los demás sistemas, la DL-valina tiene un efecto disruptor de la estructura del solvente mixto (mezclas acuosas de $\left.\mathrm{NaNO}_{3}\right)^{24}$

\begin{tabular}{|c|c|c|c|c|c|c|c|c|}
\hline$m$ valina & $\rho$ & $V_{\phi}$ & $\rho$ & $V_{\phi}$ & $\rho$ & $V_{\phi}$ & $\rho$ & $V_{\phi}$ \\
\hline $\mathrm{mol} \mathrm{kg}^{-1}$ & $\mathrm{~g} \mathrm{~cm}^{-3}$ & $\mathrm{~cm}^{3} \mathrm{~mol}^{-1}$ & $\mathrm{~g} \mathrm{~cm}^{-3}$ & $\mathrm{~cm}^{3} \mathrm{~mol}^{-1}$ & $\mathrm{~g} \mathrm{~cm}^{-3}$ & $\mathrm{~cm}^{3} \mathrm{~mol}^{-1}$ & $\mathrm{~g} \mathrm{~cm}^{-3}$ & $\mathrm{~cm}^{3} \mathrm{~mol}^{-1}$ \\
\hline \multicolumn{9}{|c|}{$m \mathrm{NaNO}_{3}=0.00000 \mathrm{~mol} \mathrm{~kg}^{-1}$} \\
\hline & \multicolumn{2}{|c|}{$283.15 \mathrm{~K}$} & \multicolumn{2}{|c|}{$288.15 \mathrm{~K}$} & \multicolumn{2}{|c|}{$293.15 \mathrm{~K}$} & \multicolumn{2}{|c|}{$298.15 \mathrm{~K}$} \\
\hline 0.00000 & 0.99969 & & 0.99909 & & 0.99819 & & 0.99704 & \\
\hline 0.01050 & 0.99989 & 98.77 & 0.99928 & 99.11 & 0.99838 & 99.37 & 0.99722 & 99.66 \\
\hline 0.02000 & 1.00017 & 93.13 & 0.99956 & 93.52 & 0.99866 & 93.83 & 0.99750 & 94.17 \\
\hline 0.03080 & 1.00049 & 91.38 & 0.99987 & 91.85 & 0.99896 & 92.34 & 0.99779 & 92.74 \\
\hline 0.05090 & 1.00103 & 90.87 & 1.00040 & 91.29 & 0.99948 & 91.83 & 0.99832 & 92.09 \\
\hline 0.07980 & 1.00180 & 90.58 & 1.00117 & 90.91 & 1.00025 & 91.30 & 0.99907 & 91.66 \\
\hline 0.09930 & 1.00233 & 90.35 & 1.00170 & 90.74 & 1.00077 & 91.08 & 0.99959 & 91.43 \\
\hline 0.15230 & 1.00376 & 90.10 & 1.00310 & 90.49 & 1.00216 & 90.84 & 1.00096 & 91.19 \\
\hline 0.20990 & 1.00533 & 89.83 & 1.00464 & 90.26 & 1.00368 & 90.62 & 1.00247 & 90.98 \\
\hline 0.24980 & 1.00641 & 89.67 & 1.00571 & 90.13 & 1.00474 & 90.47 & 1.00351 & 90.84 \\
\hline \multirow[t]{2}{*}{0.30210} & 1.00783 & 89.50 & 1.00711 & 89.93 & 1.00612 & 90.32 & 1.00488 & 90.68 \\
\hline & \multicolumn{2}{|c|}{$303.15 \mathrm{~K}$} & \multicolumn{2}{|c|}{$308.15 \mathrm{~K}$} & \multicolumn{2}{|c|}{$313.15 \mathrm{~K}$} & \multicolumn{2}{|c|}{$318.15 \mathrm{~K}$} \\
\hline 0.00000 & 0.99564 & & 0.99402 & & 0.99221 & & 0.99020 & \\
\hline 0.01050 & 0.99582 & 99.87 & 0.99421 & 99.91 & 0.99239 & 100.06 & 0.99039 & 100.13 \\
\hline 0.02000 & 0.99610 & 94.37 & 0.99448 & 94.63 & 0.99266 & 94.92 & 0.99066 & 95.17 \\
\hline 0.03080 & 0.99639 & 92.98 & 0.99477 & 93.36 & 0.99295 & 93.64 & 0.99094 & 93.92 \\
\hline 0.05090 & 0.99691 & 92.44 & 0.99528 & 92.74 & 0.99346 & 93.03 & 0.99145 & 93.27 \\
\hline 0.07980 & 0.99765 & 92.00 & 0.99603 & 92.26 & 0.99419 & 92.65 & 0.99218 & 92.89 \\
\hline 0.09930 & 0.99816 & 91.77 & 0.99653 & 92.09 & 0.99469 & 92.40 & 0.99267 & 92.71 \\
\hline 0.15230 & 0.99953 & 91.54 & 0.99788 & 91.85 & 0.99604 & 92.16 & 0.99401 & 92.45 \\
\hline 0.20990 & 1.00102 & 91.30 & 0.99936 & 91.61 & 0.99750 & 91.95 & 0.99547 & 92.20 \\
\hline 0.24980 & 1.00205 & 91.18 & 1.00039 & 91.49 & 0.99853 & 91.79 & 0.99649 & 92.05 \\
\hline 0.30210 & 1.00340 & 91.04 & 1.00173 & 91.32 & 0.99986 & 91.62 & 0.99783 & 91.86 \\
\hline \multicolumn{9}{|c|}{$m \mathrm{NaNO}_{3}=0.01064 \mathrm{~mol} \mathrm{~kg}^{-1}$} \\
\hline & \multicolumn{2}{|c|}{$283.15 \mathrm{~K}$} & \multicolumn{2}{|c|}{$288.15 \mathrm{~K}$} & \multicolumn{2}{|c|}{$293.15 \mathrm{~K}$} & \multicolumn{2}{|c|}{$298.15 \mathrm{~K}$} \\
\hline 0.00000 & 1.00056 & & 0.99995 & & 0.99905 & & 0.99788 & \\
\hline 0.05097 & 1.00192 & 90.44 & 1.00128 & 90.90 & 1.00035 & 91.39 & 0.99917 & 91.76 \\
\hline 0.08072 & 1.00272 & 90.27 & 1.00207 & 90.72 & 1.00113 & 91.19 & 0.99994 & 91.59 \\
\hline 0.09956 & 1.00322 & 90.20 & 1.00257 & 90.64 & 1.00162 & 91.11 & 1.00042 & 91.51 \\
\hline 0.20069 & 1.00592 & 89.95 & 1.00523 & 90.37 & 1.00424 & 90.86 & 1.00301 & 91.25 \\
\hline 0.25061 & 1.00724 & 89.87 & 1.00653 & 90.29 & 1.00553 & 90.76 & 1.00428 & 91.15 \\
\hline 0.30022 & 1.00856 & 89.76 & 1.00783 & 90.18 & 1.00680 & 90.67 & 1.00554 & 91.06 \\
\hline 0.35005 & 1.00987 & 89.70 & 1.00913 & 90.11 & 1.00807 & 90.60 & 1.00680 & 90.98 \\
\hline \multirow[t]{2}{*}{0.39684} & 1.01110 & 89.62 & 1.01034 & 90.05 & 1.00927 & 90.53 & 1.00798 & 90.92 \\
\hline & \multicolumn{2}{|c|}{$303.15 \mathrm{~K}$} & \multicolumn{2}{|c|}{$308.15 \mathrm{~K}$} & \multicolumn{2}{|c|}{$313.15 \mathrm{~K}$} & \multicolumn{2}{|c|}{$318.15 \mathrm{~K}$} \\
\hline 0.00000 & 0.99648 & & 0.99486 & & 0.99304 & & 0.99103 & \\
\hline 0.05097 & 0.99776 & 92.12 & 0.99612 & 92.51 & 0.99429 & 92.86 & 0.99227 & 93.28 \\
\hline 0.08072 & 0.99851 & 91.94 & 0.99687 & 92.35 & 0.99503 & 92.70 & 0.99300 & 93.10 \\
\hline 0.09956 & 0.99899 & 91.87 & 0.99734 & 92.26 & 0.99550 & 92.63 & 0.99347 & 93.03 \\
\hline
\end{tabular}

Tabla II. Densidades $\rho$ y volúmenes molares aparentes $V_{\phi}$ para la DL-valina en mezclas acuosas de $\mathrm{NaNO}_{3}$ a las temperaturas de 283.15 hasta $318.15 \mathrm{~K}$. 


\begin{tabular}{|c|c|c|c|c|c|c|c|c|}
\hline$m$ valina & $\rho$ & $V_{\phi}$ & $\rho$ & $V_{\phi}$ & $\rho$ & $V_{\phi}$ & $\rho$ & $V_{\phi}$ \\
\hline $\mathrm{mol} \mathrm{kg}^{-1}$ & $\mathrm{~g} \mathrm{~cm}^{-3}$ & $\mathrm{~cm}^{3} \mathrm{~mol}^{-1}$ & $\mathrm{~g} \mathrm{~cm}^{-3}$ & $\mathrm{~cm}^{3} \mathrm{~mol}^{-1}$ & $\mathrm{~g} \mathrm{~cm}^{-3}$ & $\mathrm{~cm}^{3} \mathrm{~mol}^{-1}$ & $\mathrm{~g} \mathrm{~cm}^{-3}$ & $\mathrm{~cm}^{3} \mathrm{~mol}^{-1}$ \\
\hline 0.20069 & 1.00155 & 91.62 & 0.99988 & 91.98 & 0.99801 & 92.36 & 0.99596 & 92.74 \\
\hline 0.25061 & 1.00281 & 91.54 & 1.00114 & 91.86 & 0.99925 & 92.25 & 0.99718 & 92.64 \\
\hline 0.30022 & 1.00406 & 91.44 & 1.00237 & 91.78 & 1.00047 & 92.16 & 0.99839 & 92.56 \\
\hline 0.35005 & 1.00530 & 91.36 & 1.00361 & 91.69 & 1.00170 & 92.07 & 0.99960 & 92.47 \\
\hline 0.39684 & 1.00647 & 91.28 & 1.00476 & 91.64 & 1.00284 & 92.02 & 1.00073 & 92.40 \\
\hline \multicolumn{9}{|c|}{$m \mathrm{NaNO}_{3}=0.04132 \mathrm{~mol} \mathrm{~kg}^{-1}$} \\
\hline & \multicolumn{2}{|c|}{$283.15 \mathrm{~K}$} & \multicolumn{2}{|c|}{$288.15 \mathrm{~K}$} & \multicolumn{2}{|c|}{$293.15 \mathrm{~K}$} & \multicolumn{2}{|c|}{$298.15 \mathrm{~K}$} \\
\hline 0.00000 & 1.00231 & & 1.00166 & & 1.00073 & & 0.99954 & \\
\hline 0.05038 & 1.00365 & 90.44 & 1.00298 & 90.81 & 1.00202 & 91.26 & 1.00082 & 91.64 \\
\hline 0.07998 & 1.00443 & 90.30 & 1.00376 & 90.70 & 1.00279 & 91.14 & 1.00157 & 91.52 \\
\hline 0.09896 & 1.00494 & 90.24 & 1.00425 & 90.63 & 1.00328 & 91.07 & 1.00206 & 91.46 \\
\hline 0.20069 & 1.00763 & 90.02 & 1.00691 & 90.43 & 1.00590 & 90.88 & 1.00464 & 91.27 \\
\hline 0.25093 & 1.00895 & 89.95 & 1.00821 & 90.37 & 1.00718 & 90.81 & 1.00591 & 91.20 \\
\hline 0.29985 & 1.01023 & 89.89 & 1.00947 & 90.31 & 1.00843 & 90.73 & 1.00714 & 91.13 \\
\hline 0.34962 & 1.01152 & 89.83 & 1.01075 & 90.24 & 1.00968 & 90.68 & 1.00838 & 91.08 \\
\hline \multirow[t]{2}{*}{0.40049} & 1.01284 & 89.76 & 1.01205 & 90.17 & 1.01096 & 90.62 & 1.00964 & 91.03 \\
\hline & \multicolumn{2}{|c|}{$303.15 \mathrm{~K}$} & \multicolumn{2}{|c|}{$308.15 \mathrm{~K}$} & \multicolumn{2}{|c|}{$313.15 \mathrm{~K}$} & \multicolumn{2}{|c|}{$318.15 \mathrm{~K}$} \\
\hline 0.00000 & 0.99811 & & 0.99647 & & 0.99463 & & 0.99261 & \\
\hline 0.05038 & 0.99937 & 92.07 & 0.99773 & 92.36 & 0.99588 & 92.76 & 0.99384 & 93.09 \\
\hline 0.07998 & 1.00012 & 91.93 & 0.99847 & 92.24 & 0.99661 & 92.65 & 0.99457 & 92.94 \\
\hline 0.09896 & 1.00060 & 91.85 & 0.99894 & 92.17 & 0.99708 & 92.58 & 0.99504 & 92.86 \\
\hline 0.20069 & 1.00317 & 91.63 & 1.00148 & 91.96 & 0.99958 & 92.39 & 0.99753 & 92.69 \\
\hline 0.25093 & 1.00442 & 91.54 & 1.00273 & 91.89 & 1.00081 & 92.33 & 0.99875 & 92.63 \\
\hline 0.29985 & 1.00564 & 91.47 & 1.00393 & 91.82 & 1.00200 & 92.27 & 0.99993 & 92.57 \\
\hline 0.34962 & 1.00687 & 91.42 & 1.00515 & 91.76 & 1.00320 & 92.22 & 1.00113 & 92.51 \\
\hline 0.40049 & 1.00813 & 91.35 & 1.00639 & 91.72 & 1.00443 & 92.16 & 1.00235 & 92.45 \\
\hline \multicolumn{9}{|c|}{$m \mathrm{NaNO}_{3}=0.10086 \mathrm{~mol} \mathrm{~kg}^{-1}$} \\
\hline & \multicolumn{2}{|c|}{$283.15 \mathrm{~K}$} & \multicolumn{2}{|c|}{$288.15^{3} \mathrm{~K}$} & \multicolumn{2}{|c|}{$293.15 \mathrm{~K}$} & \multicolumn{2}{|c|}{$298.15 \mathrm{~K}$} \\
\hline 0.00000 & 1.00580 & & 1.00509 & & 1.00409 & & 1.00285 & \\
\hline 0.05034 & 1.00714 & 90.02 & 1.00641 & 90.39 & 1.00540 & 90.79 & 1.00414 & 91.21 \\
\hline 0.08080 & 1.00795 & 89.91 & 1.00721 & 90.30 & 1.00619 & 90.69 & 1.00492 & 91.10 \\
\hline 0.10084 & 1.00849 & 89.87 & 1.00774 & 90.27 & 1.00671 & 90.64 & 1.00544 & 91.06 \\
\hline 0.20070 & 1.01113 & 89.72 & 1.01034 & 90.14 & 1.00929 & 90.50 & 1.00799 & 90.90 \\
\hline 0.25102 & 1.01245 & 89.67 & 1.01165 & 90.10 & 1.01058 & 90.45 & 1.00926 & 90.85 \\
\hline 0.29998 & 1.01374 & 89.62 & 1.01290 & 90.05 & 1.01183 & 90.40 & 1.01049 & 90.79 \\
\hline 0.34969 & 1.01502 & 89.58 & 1.01418 & 90.01 & 1.01309 & 90.35 & 1.01173 & 90.76 \\
\hline \multirow[t]{2}{*}{0.39866} & 1.01629 & 89.52 & 1.01542 & 89.96 & 1.01432 & 90.31 & 1.01295 & 90.70 \\
\hline & \multicolumn{2}{|c|}{$303.15 \mathrm{~K}$} & \multicolumn{2}{|c|}{$308.15 \mathrm{~K}$} & \multicolumn{2}{|c|}{$313.15 \mathrm{~K}$} & \multicolumn{2}{|c|}{$318.15 \mathrm{~K}$} \\
\hline 0.00000 & 1.00138 & & 0.99970 & & 0.99782 & & 0.99560 & \\
\hline 0.05034 & 1.00266 & 91.56 & 1.00096 & 91.98 & 0.99907 & 92.30 & 0.99684 & 92.64 \\
\hline 0.08080 & 1.00343 & 91.47 & 1.00173 & 91.87 & 0.99984 & 92.18 & 0.99760 & 92.52 \\
\hline 0.10084 & 1.00394 & 91.42 & 1.00223 & 91.83 & 1.00034 & 92.13 & 0.99810 & 92.47 \\
\hline 0.20070 & 1.00646 & 91.29 & 1.00473 & 91.67 & 1.00281 & 91.98 & 1.00056 & 92.27 \\
\hline 0.25102 & 1.00772 & 91.23 & 1.00597 & 91.62 & 1.00405 & 91.92 & 1.00179 & 92.21 \\
\hline 0.29998 & 1.00894 & 91.18 & 1.00718 & 91.57 & 1.00524 & 91.88 & 1.00298 & 92.16 \\
\hline 0.34969 & 1.01016 & 91.14 & 1.00839 & 91.52 & 1.00645 & 91.82 & 1.00419 & 92.10 \\
\hline 0.39866 & 1.01136 & 91.11 & 1.00958 & 91.48 & 1.00764 & 91.78 & 1.00536 & 92.05 \\
\hline
\end{tabular}




\begin{tabular}{|c|c|c|c|c|c|c|c|c|}
\hline$m$ valina & $\rho$ & $V_{\phi}$ & $\rho$ & $V_{\phi}$ & $\rho$ & $V_{\phi}$ & $\rho$ & $V_{\phi}$ \\
\hline $\mathrm{mol} \mathrm{kg}^{-1}$ & $\mathrm{~g} \mathrm{~cm}^{-3}$ & $\mathrm{~cm}^{3} \mathrm{~mol}^{-1}$ & $\mathrm{~g} \mathrm{~cm}^{-3}$ & $\mathrm{~cm}^{3} \mathrm{~mol}^{-1}$ & $\mathrm{~g} \mathrm{~cm}^{-3}$ & $\mathrm{~cm}^{3} \mathrm{~mol}^{-1}$ & $\mathrm{~g} \mathrm{~cm}^{-3}$ & $\mathrm{~cm}^{3} \mathrm{~mol}^{-1}$ \\
\hline \multicolumn{9}{|c|}{$m \mathrm{NaNO}_{3}=0.40131 \mathrm{~mol} \mathrm{~kg}^{-1}$} \\
\hline & \multicolumn{2}{|c|}{$283.15 \mathrm{~K}$} & \multicolumn{2}{|c|}{$288.15 \mathrm{~K}$} & \multicolumn{2}{|c|}{$293.15 \mathrm{~K}$} & \multicolumn{2}{|c|}{$298.15 \mathrm{~K}$} \\
\hline 0.00000 & 1.02287 & & 1.02185 & & 1.02059 & & 1.01912 & \\
\hline 0.05034 & 1.02419 & 89.33 & 1.02315 & 89.72 & 1.02189 & 90.00 & 1.02040 & 90.32 \\
\hline 0.08028 & 1.02498 & 89.26 & 1.02393 & 89.66 & 1.02265 & 89.94 & 1.02116 & 90.26 \\
\hline 0.10084 & 1.02551 & 89.24 & 1.02446 & 89.62 & 1.02318 & 89.91 & 1.02168 & 90.23 \\
\hline 0.20044 & 1.02809 & 89.16 & 1.02701 & 89.54 & 1.02571 & 89.84 & 1.02419 & 90.14 \\
\hline 0.25074 & 1.02939 & 89.12 & 1.02828 & 89.51 & 1.02697 & 89.81 & 1.02544 & 90.10 \\
\hline 0.29995 & 1.03064 & 89.09 & 1.02952 & 89.48 & 1.02820 & 89.78 & 1.02666 & 90.07 \\
\hline 0.35005 & 1.03191 & 89.06 & 1.03077 & 89.46 & 1.02944 & 89.75 & 1.02789 & 90.04 \\
\hline \multirow[t]{2}{*}{0.39786} & 1.03311 & 89.04 & 1.03196 & 89.43 & 1.03061 & 89.73 & 1.02906 & 90.01 \\
\hline & \multicolumn{2}{|c|}{$303.15 \mathrm{~K}$} & \multicolumn{2}{|c|}{$308.15 \mathrm{~K}$} & \multicolumn{2}{|c|}{$313.15 \mathrm{~K}$} & \multicolumn{2}{|c|}{$318.15 \mathrm{~K}$} \\
\hline 0.00000 & 1.01744 & & 1.01558 & & 1.01354 & & 1.01130 & \\
\hline 0.05034 & 1.01871 & 90.71 & 1.01684 & 91.03 & 1.01479 & 91.35 & 1.01254 & 91.64 \\
\hline 0.08028 & 1.01946 & 90.64 & 1.01758 & 90.97 & 1.01553 & 91.27 & 1.01328 & 91.54 \\
\hline 0.10084 & 1.01998 & 90.60 & 1.01810 & 90.93 & 1.01604 & 91.23 & 1.01379 & 91.50 \\
\hline 0.20044 & 1.02246 & 90.50 & 1.02056 & 90.81 & 1.01850 & 91.08 & 1.01623 & 91.33 \\
\hline 0.25074 & 1.02370 & 90.45 & 1.02179 & 90.78 & 1.01972 & 91.04 & 1.01745 & 91.29 \\
\hline 0.29995 & 1.02491 & 90.42 & 1.02299 & 90.74 & 1.02091 & 91.01 & 1.01864 & 91.26 \\
\hline 0.35005 & 1.02613 & 90.39 & 1.02420 & 90.71 & 1.02212 & 90.97 & 1.01984 & 91.22 \\
\hline 0.39786 & 1.02728 & 90.37 & 1.02534 & 90.69 & 1.02325 & 90.95 & 1.02098 & 91.19 \\
\hline \multicolumn{9}{|c|}{$m \mathrm{NaNO}_{3}=0.90145 \mathrm{~mol} \mathrm{~kg}^{-1}$} \\
\hline & \multicolumn{2}{|c|}{$283.15 \mathrm{~K}$} & \multicolumn{2}{|c|}{$288.15 \mathrm{~K}$} & \multicolumn{2}{|c|}{$293.15 \mathrm{~K}$} & \multicolumn{2}{|c|}{$298.15 \mathrm{~K}$} \\
\hline 0.00000 & 1.05001 & & 1.04857 & & 1.04694 & & 1.04513 & \\
\hline 0.05002 & 1.05132 & 87.87 & 1.04986 & 88.24 & 1.04821 & 88.64 & 1.04639 & 88.97 \\
\hline 0.08010 & 1.05209 & 87.84 & 1.05063 & 88.20 & 1.04897 & 88.60 & 1.04714 & 88.93 \\
\hline 0.09997 & 1.05261 & 87.82 & 1.05114 & 88.18 & 1.04947 & 88.58 & 1.04764 & 88.91 \\
\hline 0.20072 & 1.05518 & 87.78 & 1.05369 & 88.12 & 1.05199 & 88.54 & 1.05013 & 88.86 \\
\hline 0.25066 & 1.05645 & 87.76 & 1.05494 & 88.10 & 1.05322 & 88.52 & 1.05135 & 88.84 \\
\hline 0.30012 & 1.05769 & 87.74 & 1.05616 & 88.08 & 1.05443 & 88.50 & 1.05255 & 88.82 \\
\hline 0.35005 & 1.05893 & 87.73 & 1.05739 & 88.07 & 1.05564 & 88.49 & 1.05375 & 88.80 \\
\hline \multirow[t]{2}{*}{0.40188} & 1.06021 & 87.71 & 1.05866 & 88.05 & 1.05689 & 88.47 & 1.05499 & 88.79 \\
\hline & \multicolumn{2}{|c|}{$303.15 \mathrm{~K}$} & \multicolumn{2}{|c|}{$308.15 \mathrm{~K}$} & \multicolumn{2}{|c|}{$313.15 \mathrm{~K}$} & \multicolumn{2}{|c|}{$318.15 \mathrm{~K}$} \\
\hline 0.00000 & 1.04315 & & 1.04102 & & 1.03862 & & 1.03587 & \\
\hline 0.05002 & 1.04440 & 89.32 & 1.04225 & 89.62 & 1.03985 & 89.95 & 1.03709 & 90.25 \\
\hline 0.08010 & 1.04514 & 89.29 & 1.04299 & 89.59 & 1.04059 & 89.91 & 1.03782 & 90.23 \\
\hline 0.09997 & 1.04563 & 89.27 & 1.04348 & 89.58 & 1.04107 & 89.90 & 1.03831 & 90.21 \\
\hline 0.20072 & 1.04810 & 89.22 & 1.04593 & 89.53 & 1.04350 & 89.83 & 1.04072 & 90.16 \\
\hline 0.25066 & 1.04931 & 89.20 & 1.04713 & 89.51 & 1.04469 & 89.81 & 1.04190 & 90.15 \\
\hline 0.30012 & 1.05049 & 89.19 & 1.04831 & 89.49 & 1.04587 & 89.79 & 1.04307 & 90.13 \\
\hline 0.35005 & 1.05168 & 89.17 & 1.04949 & 89.47 & 1.04704 & 89.78 & 1.04423 & 90.11 \\
\hline 0.40188 & 1.05291 & 89.15 & 1.05070 & 89.46 & 1.04825 & 89.76 & 1.04543 & 90.10 \\
\hline
\end{tabular}

Tabla II. Densidades $\rho$ y volúmenes molares aparentes $V_{\phi}$ para la DL-valina en mezclas acuosas de $\mathrm{NaNO}_{3}$ a las temperaturas de 283.15 hasta $318.15 \mathrm{~K}$ (continuación). 


\begin{tabular}{|c|c|c|c|}
\hline $\begin{array}{c}m \mathrm{NaNO}_{3} / \\
\mathrm{mol} \mathrm{kg}^{-1}\end{array}$ & $V_{\phi}^{0} / \mathrm{cm}^{3} \mathrm{~mol}^{-1}$ & $\begin{array}{c}S_{V} / \mathrm{cm}^{3} \mathrm{~mol}^{-2} \\
\mathrm{~kg}\end{array}$ & $\mathrm{R}^{2}$ \\
\hline \multicolumn{4}{|c|}{$283.15 \mathrm{~K}$} \\
\hline 0.00000 & 91.53 & -3.69 & 0.9992 \\
\hline 0.01064 & 90.77 & -1.83 & 0.9949 \\
\hline 0.04132 & 90.65 & -1.39 & 0.9970 \\
\hline 0.10086 & 90.20 & -1.06 & 0.9960 \\
\hline 0.40131 & 89.45 & -0.65 & 0.9963 \\
\hline 0.90135 & 87.94 & -0.36 & 0.9983 \\
\hline \multicolumn{4}{|c|}{$288.15 \mathrm{~K}$} \\
\hline 0.00000 & 91.82 & -3.42 & 0.9989 \\
\hline 0.01064 & 91.19 & -1.82 & 0.9965 \\
\hline 0.04132 & 91.06 & -1.39 & 0.9949 \\
\hline 0.10086 & 90.60 & -1.01 & 0.9963 \\
\hline 0.40131 & 89.81 & -0.61 & 0.9968 \\
\hline 0.90135 & 88.29 & -0.38 & 0.9984 \\
\hline \multicolumn{4}{|c|}{$293.15 \mathrm{~K}$} \\
\hline 0.00000 & 92.10 & -3.24 & 0.9996 \\
\hline 0.01064 & 91.66 & -1.80 & 0.9994 \\
\hline 0.04132 & 91.49 & -1.37 & 0.9979 \\
\hline 0.10086 & 90.97 & -1.04 & 0.9985 \\
\hline 0.40131 & 90.12 & -0.62 & 0.9962 \\
\hline 0.90135 & 88.69 & -0.35 & 0.9985 \\
\hline \multicolumn{4}{|c|}{$298.15 \mathrm{~K}$} \\
\hline 0.00000 & 92.44 & -3.20 & 0.9999 \\
\hline 0.01064 & 92.07 & -1.84 & 0.9981 \\
\hline 0.04132 & 91.86 & -1.32 & 0.9987 \\
\hline 0.10086 & 91.38 & -1.07 & 0.9932 \\
\hline 0.40131 & 90.45 & -0.69 & 0.9990 \\
\hline 0.90135 & 89.04 & -0.40 & 0.9982 \\
\hline \multicolumn{4}{|c|}{$303.15 \mathrm{~K}$} \\
\hline 0.00000 & 92.77 & -3.17 & 0.9987 \\
\hline 0.01064 & 92.46 & -1.87 & 0.9969 \\
\hline 0.04132 & 92.28 & -1.46 & 0.9981 \\
\hline 0.10086 & 91.74 & -1.01 & 0.9981 \\
\hline 0.40131 & 90.81 & -0.71 & 0.9928 \\
\hline 0.90135 & 89.39 & -0.37 & 0.9966 \\
\hline \multicolumn{4}{|c|}{308.15 K } \\
\hline 0.00000 & 93.11 & -3.26 & 0.9998 \\
\hline 0.01064 & 92.81 & -1.88 & 0.9994 \\
\hline 0.04132 & 92.55 & -1.32 & 0.9952 \\
\hline 0.10086 & 92.16 & -1.08 & 0.9996 \\
\hline 0.40131 & 91.12 & -0.69 & 0.9998 \\
\hline 0.90135 & 89.69 & -0.37 & 0.9977 \\
\hline \multicolumn{4}{|c|}{$313.15 \mathrm{~K}$} \\
\hline 0.00000 & 93.45 & -3.32 & 0.9992 \\
\hline 0.01064 & 93.20 & -1.90 & 0.9957 \\
\hline
\end{tabular}

Tabla III. Volúmenes molares aparentes a dilución infinita $V_{\phi}^{0}$ para la DL-valina en mezclas acuosas de $\mathrm{NaNO}_{3}$ a las temperaturas de 283.15, 288.15, 293.15, 298.15, 303.15, 308.15, 313.15 y $318.15 \mathrm{~K}$

\begin{tabular}{|c|c|c|c|}
\hline $\begin{array}{c}m \mathrm{NaNO}_{3} / \\
\mathrm{mol} \mathrm{kg}^{-1}\end{array}$ & $V_{\phi}^{0} / \mathrm{cm}^{3} \mathrm{~mol}^{-1}$ & $\begin{array}{c}S_{V} / \mathrm{cm}^{3} \mathrm{~mol}^{-2} \\
\mathrm{~kg}\end{array}$ & $\mathrm{R}^{2}$ \\
\hline 0.04132 & 92.95 & -1.25 & 0.9980 \\
\hline 0.10086 & 92.48 & -1.10 & 0.9982 \\
\hline 0.40131 & 91.41 & -0.72 & 0.9970 \\
\hline 0.90135 & 90.01 & -0.39 & 0.9988 \\
\hline \multicolumn{4}{|c|}{$\mathbf{3 1 8 . 1 5 ~ K}$} \\
\hline 0.00000 & 93.85 & -3.74 & 0.9996 \\
\hline 0.01064 & 93.58 & -1.87 & 0.9990 \\
\hline 0.04132 & 93.29 & -1.32 & 0.9974 \\
\hline 0.10086 & 92.80 & -1.18 & 0.9992 \\
\hline 0.40131 & 91.66 & -0.75 & 0.9984 \\
\hline 0.90135 & 90.32 & -0.34 & 0.9993 \\
\hline
\end{tabular}

Tabla III. Volúmenes molares aparentes a dilución infinita $V_{\phi}^{0}$ para la DL-valina en mezclas acuosas de $\mathrm{NaNO}_{3}$ a las temperaturas de 283.15, 288.15, 293.15, 298.15, 303.15, 308.15, 313.15 y $318.15 \mathrm{~K}$ (continuación).

Para examinar las interacciones moleculares predominantes en solución se determinaron los volúmenes molares parciales de transferencia a dilución infinita $\Delta V_{\phi}^{0}$, que fueron obtenidos con la ecuación (6) y los resultados se presentan en la Tabla V.

$\Delta V_{\phi}^{0}=V_{\phi}^{0}\left(\right.$ en mezclas acuosas de $\left.\mathrm{NaNO}_{3}\right)-V_{\phi}^{0}($ en agua $)$

Los valores negativos obtenidos para $\Delta V_{\phi}^{0}$ de la DL-valina en las mezclas acuosas de $\mathrm{NaNO}_{3}$ podrían explicarse con ayuda del modelo de superposición de coesferas hidratadas ${ }^{25}$ y del concepto de que en el estado de dilución infinita las interacciones soluto-soluto están ausentes ${ }^{26-27}$. Además, de acuerdo con Mishra y Ahluwalia, los valores negativos de los volúmenes de transferencia obtenidos en este trabajo son el resultado del predominio en solución de la interacción solutocosolvente: ion-hidrofóbica, que ocurre entre el grupo isopropil $-\mathrm{CH}\left(\mathrm{CH}_{3}\right)_{2}$ del aminoácido DL-valina y los iones $\mathrm{Na}^{+} \mathrm{y} \mathrm{NO}_{3}{ }^{-}$del cosolvente ${ }^{8}$. Mientras que los valores negativos para $\Delta V_{\phi}^{0}$ son el resultado del efecto que pudiera tener el nitrato de sodio sobre las moléculas de agua en las coesferas de hidratación y sobre la molécula de soluto ${ }^{28}$.

\begin{tabular}{|c|c|}
\hline $\begin{array}{c}m \mathrm{NaNO}_{3} / \mathrm{mol} \mathrm{kg}^{-1} \\
\text { en solución acuosa }\end{array}$ & $\partial^{2} V_{\phi}^{0} / \partial T^{2}$ \\
\hline 0.00000 & $0.00058 \pm 0.00004$ \\
\hline 0.01064 & $-0.00059 \pm 0.00007$ \\
\hline 0.04132 & $-0.00061 \pm 0.00009$ \\
\hline 0.10086 & $-0.00045 \pm 0.00007$ \\
\hline 0.40131 & $-0.00049 \pm 0.00007$ \\
\hline 0.90135 & $-0.00058 \pm 0.00004$ \\
\hline
\end{tabular}

Tabla IV. Valor de $\partial^{2} V_{\phi}^{0} / \partial T^{2}$ de la DL-valina en mezclas acuosas de $\mathrm{NaNO}_{3}$ a diferentes temperaturas. 
Consecuentemente, el predomino de dichas interacciones produce un aumento en el número de moléculas de agua liberada desde la esfera de solvatación hacia la fase voluminosa, produciéndose así una disrupción local en la estructura del solvente ${ }^{29-30}$, este resultado es consistente con el criterio de la segunda derivada del volumen molar a dilución infinita con respecto a la temperatura, como lo sugiere el análisis de $\partial^{2} V_{\phi}^{0} / \partial T^{2}$

Por otra parte, los valores negativos obtenidos para $\Delta V_{\phi}^{0}$ pueden ser también explicados con ayuda de la ecuación (7) propuesta por Shahidi y colaboradores ${ }^{31}$.

$\Delta V_{\phi}^{0}=\Delta V_{V W}+\Delta V_{V}+\Delta V_{S}$

En esta construcción, los volúmenes molares parciales a dilución infinita son consecuencia de las siguientes contribuciones: $\Delta V_{V W}$ que es el volumen de van der Waals, $\Delta V_{V}$ es el volumen asociado a los espacios vacíos en solución y $\Delta V_{S}$ es el volumen de contracción debido al efecto de electrostricción. Asumiendo que $\Delta V_{V W}$ y $\Delta V_{V}$ tienen la misma magnitud en agua que en soluciones acuosas, es decir son nulos, se deduce que cualquier variación de los volúmenes de transferencia es sólo consecuencia del efecto de electrostricción $V_{S}{ }^{29}$. El signo negativo para $\Delta V_{\phi^{3}}^{0}$ indica una disminución en el volumen a causa del efecto de electrostricción.

Esta situación nos advierte, que la presencia del nitrato de sodio en el sistema (DL-valina + mezclas acuosas de $\mathrm{NaNO}_{3}$ ) aumenta el efecto de electrostricción alrededor de la DL-valina, como lo reflejan los números de hidratación, que se obtienen a continuación:

Los números de hidratación $N_{H}$ para la DL-valina a cada temperatura y concentración de $\mathrm{NaNO}_{3}$ se calcularon con ayuda del modelo propuesto por Millero ${ }^{32}$, a partir del volumen molar parcial del aminoácido, mediante la ecuación (8).

$\bar{V}_{2}^{0}=\bar{V}_{2}^{0}($ int $)+\bar{V}_{2}^{0}($ elect $)$
Donde $\bar{V}_{2}^{0}$ es el volumen molar parcial a dilución infinita de la DL-valina obtenido experimentalmente, $\bar{V}_{2}^{0}$ (int) es el volumen molar parcial intrínseco de la DL-valina, que se puede expresar como la adición del volumen de van der Waals y el volumen debido al efecto de empaquetamiento ${ }^{33}, \bar{V}_{2}^{0}$ (elect) es el volumen molar parcial de electrostricción debido a la hidratación del aminoácido. Los valores de $\bar{V}_{2}^{0}$ (int) pueden ser obtenidos del volumen molar de un cristal de la DL-valina utilizando la ecuación (9).

$\bar{V}_{2}^{0}($ int $)=\left(\frac{0.7}{0.634}\right) \bar{V}_{2}^{0}($ cristal $)$

Donde $\bar{V}_{2}^{0}$ (cristal) es el volumen molar de los cristales del aminoácido, y se obtiene dividiendo la masa molecular de la DL-valina entre su densidad en estado puro ${ }^{34}$. Esta ecuación fue originalmente usada por Millero, para evaluar el comportamiento de aminoácidos en agua; sin embargo, también ha sido usada por otros investigadores en solventes acuosos mixtos ${ }^{35-40}$. Una vez conocido el volumen de electrostricción es posible determinar la cantidad relativa de moléculas de agua alrededor del aminoácido hidratado utilizando la siguiente ecuación:

$\bar{V}_{2}^{0}($ elect $)=N_{H}\left(\bar{V}_{E}^{0}-\bar{V}_{B}^{0}\right)$

En este modelo se asume que por cada molécula de agua que pase desde la fase voluminosa hasta la región cercana al aminoácido el volumen decrece en $\left(\bar{V}_{E}^{0}-\bar{V}_{B}^{0}\right)$. En este trabajo, para evaluar el término $\left(\bar{V}_{E}^{0}-\bar{V}_{B}^{0}\right)$ fueron usados los valores de: $-2.9,-3.3$, $-4.0 \mathrm{~cm}^{3} \mathrm{~mol}^{-1}$ a $288.15,298.15$ y $308.15 \mathrm{~K}$ reportados por Yan y colaboradores ${ }^{4}$, en adición a los extrapolados por Páez y colaboradores ${ }^{36} \mathrm{de}:-2.7,-3.1,-3.6,-4.6 \mathrm{y}-5.5 \mathrm{~cm}^{3} \mathrm{~mol}^{-1}$ para las temperaturas de $283.15,293.15,303.15,313.15 \mathrm{y}$ 318.15 K. Finalmente los números de hidratación pueden ser obtenidos como una aproximación utilizando la ecuación (10) y los resultados se muestran en la Tabla VI.

Es evidente que los números de hidratación $N_{H}$ de la DL-valina en las mezclas acuosas de $\mathrm{NaNO}_{3}$ aumentan conforme aumenta

\begin{tabular}{|c|c|c|c|c|c|}
\hline$m \mathrm{NaNO}_{3} / \mathrm{mol} \mathrm{kg}^{-1}$ & 0.01064 & 0.04132 & 0.10086 & 0.40131 & 0.90135 \\
\hline$T / \mathrm{K}$ & \multicolumn{5}{|c|}{$\Delta V_{\phi}^{0} / \mathrm{cm}^{3} \mathrm{~mol}^{-1}$} \\
\hline 283.15 & -0.75 & -0.88 & -1.33 & -2.08 & -3.59 \\
\hline 288.15 & -0.63 & -0.76 & -1.22 & -2.01 & -3.53 \\
\hline 293.15 & -0.44 & -0.61 & -1.13 & -1.98 & -3.41 \\
\hline 298.15 & -0.37 & -0.58 & -1.06 & -2.00 & -3.40 \\
\hline 303.15 & -0.31 & -0.49 & -1.03 & -1.96 & -3.38 \\
\hline 313.15 & -0.25 & -0.50 & -0.98 & -2.05 & -3.44 \\
\hline 318.15 & -0.27 & -0.57 & -1.05 & -2.19 & -3.54 \\
\hline
\end{tabular}

Tabla V. Volúmenes molares parciales de transferencia a dilución infinita $\Delta V_{\phi}^{0}$ para la DL-valina en agua hasta las mezclas acuosas de $\mathrm{NaNO}_{3}$ 


\begin{tabular}{|c|c|c|c|c|c|c|}
\hline $\begin{array}{c}m \mathrm{NaNO}_{3} / \mathrm{mol} \\
\mathrm{kg}^{-1}\end{array}$ & 0.01064 & 0.04132 & 0.10086 & 0.40131 & 0.90135 & 0.01064 \\
\hline$T / \mathrm{K}$ & \multicolumn{7}{|c|}{$N_{H}$} \\
\hline 283.15 & 2.50 & 2.78 & 2.83 & 3.00 & 3.27 & 3.83 \\
\hline 288.15 & 2.23 & 2.45 & 2.49 & 2.65 & 2.92 & 3.45 \\
\hline 293.15 & 2.00 & 2.14 & 2.19 & 2.36 & 2.63 & 3.10 \\
\hline 298.15 & 1.77 & 1.88 & 1.95 & 2.09 & 2.38 & 2.80 \\
\hline 303.15 & 1.53 & 1.62 & 1.67 & 1.82 & 2.08 & 2.47 \\
\hline 308.15 & 1.29 & 1.37 & 1.43 & 1.53 & 1.79 & 2.15 \\
\hline 313.15 & 1.05 & 1.10 & 1.16 & 1.26 & 1.50 & 1.80 \\
\hline 318.15 & 0.81 & 0.86 & 0.92 & 1.01 & 1.22 & 1.46 \\
\hline
\end{tabular}

Tabla VI. Números de hidratación $\mathrm{N}_{H}$ para la DL-valina en mezclas acuosas de $\mathrm{NaNO}_{3}$ a diferentes temperaturas.

la concentración del cosolvente y disminuyen con el aumento de la temperatura. Este hecho sugiere que el aumento de las interacciones entre la DL-valina y los iones $\mathrm{Na}^{+}$y $\mathrm{NO}_{3}^{-}$del cosolvente generan un aumento en el efecto de electrostricción sobre las moléculas de agua, mientras que el aumento de la temperatura provoca una deshidratación de las especies solvatadas ${ }^{41}$.

\section{Conclusiones}

En este trabajo se obtuvieron valores de densidad no reportados para el sistema de DL-valina en mezclas acuosas de $\mathrm{NaNO}_{3}$ a las temperaturas desde $283.15 \mathrm{~K}$ hasta $318.15 \mathrm{~K}$ a intervalos de $5 \mathrm{~K}$. A partir de los datos experimentales de densidad obtenidos se calcularon propiedades volumétricas encontrándose que los volúmenes molares aparentes a dilución infinita $V_{\phi}^{0}$ resultaron positivos y disminuyen con el aumento de la concentración del $\mathrm{NaNO}_{3}$ e incrementan con el aumento de la temperatura. La segunda derivada de $V_{\phi}^{0}$ con respecto a la temperatura muestra que la DL-valina se comporta como un soluto disruptor de la estructura del solvente mixto (mezclas acuosas de $\mathrm{NaNO}_{3}$ ). Los volúmenes molares parciales de transferencia a dilución infinita $\Delta V_{\phi}^{0}$ indican que las interacciones dominantes se establecen entre los iones $\mathrm{Na}^{+}$y $\mathrm{NO}_{3}^{-}$del cosolvente y la parte hidrofóbica del aminoácido, generando un aumento en la contracción del volumen a causa del efecto de electrostricción. Finalmente, los resultados de los números de hidratación $N_{H}$ utilizando el método de Millero, muestran un efecto de deshidratación a medida que se incrementan los valores de temperatura; $\sin$ embargo, este parámetro no mostró sensibilidad a los cambios en los valores de la concentración del $\mathrm{NaNO}_{3}$ en las mezclas.

\section{Agradecimientos}

Los autores agradecen a la Universidad de Córdoba por el apoyo brindado para la realización de este trabajo.

\section{Referencias}

1. Yan, Z., Wang, J., Zhang, H. \& Xuan, X. Volumetric and Viscosity Properties of $\alpha$-Amino Acids and Their Groups in Aqueous Sodium Caproate Solutions. J. Chem. Eng. Data 50, 1864-1870 (2005). DOI: 10.1021/je0501484

2. Kirkwood, J. G. Theoretical Studies upon Dipolar Ions. Chem.Rev. 24,233-251 (1939). DOI: 10.1021/cr60078a004

3. Hvidt, A. \& Westh, P. Different Views on the Stability of Protein Conformations and Hydrophobic Effects. J. Solut. Chem.27,395-402(1998).DOI: 10.1023/A:1022696404041

4. Yan, Z., Wang, J., Kong, W. \& Lu, J. Effect of temperature on volumetric and viscosity properties of some $\alpha$-amino acids in aqueous calcium chloride solutions. Fluid Phase Equilibria 215, 143-150 (2004). DOI: 10.1016/j.fluid.2003.07.001

5. Dhondge, S. S., Zodape, S. P. \& Parwate, D. V. Volumetric and viscometric studies of some drugs in aqueous solutions at different temperatures. J. Chem. Thermodyn. 48, 207-212 (2012). DOI: 10.1016/j.jct.2011.12.022

6. Schrier, M. Y., Ying, A. H. C., Ross, M. E. \& Schrier, E. E. Free energy changes and structural consequences for the transfer of urea from water and ribonuclease A from dilute buffer to aqueous salt solutions. J. Phys. Chem. 81, 674-679 (1977). DOI: 10.1021/j100522a018

7. Lapanje, S., Škerjanc, J., Glavnik, S. \& Žibret, S. Thermodynamic studies of the interactions of guanidinium chloride and urea with some oligoglycines and oligoleucines. J. Chem. Thermodyn. 10, 425-433 (1978). DOI: 10.1016/0021-9614(78)90089-7

8. Mishra,A.K. \& Ahluwalia, J.C. Apparent molal volumes of amino acids, $\mathrm{N}$-acetylamino acids, and peptides in aqueous solutions. J. Phys. Chem. 88, 86-92 (1984). DOI: 10.1021/ j150645a 021

9. Bhat, R. \& Ahluwalia, J. C. Partial molar heat capacities and volumes of transfer of some amino acids and peptides from water to aqueous sodium chloride solutions at 298.15 
K. J. Phys. Chem. 89, 1099-1105 (1985). DOI: 10.1021/ j100253a011

10. Singh, S. K. \& Kishore, N. Partial Molar Volumes of Amino Acids and Peptides in Aqueous Salt Solutions at $25^{\circ} \mathrm{C}$ and a Correlation with Stability of Proteins in the Presence of Salts. J. Solut. Chem. 32, 117-135 (2003). DOI: 10.1023/A:1022946105467

11. Banipal, T. S. \& Singh, G. Thermodynamic study of solvation of some amino acids, diglycine and lysozyme in aqueous and mixed aqueous solutions. Thermochim. Acta 412, 63-83 (2004). DOI: 10.1016/j.tca.2003.08.026

12. Pal,A. \& Chauhan, N. Volumetric, viscometric, and acoustic behaviour of diglycine in aqueous saccharide solutions at different temperatures. J. Mol. Liq. 149, 29-36 (2009). DOI: 10.1016/j.molliq.2009.07.014

13. Enea, O. \& Jolicoeur, C. Heat capacities and volumes of several oligopeptides in urea-water mixtures at 25.degree.C. Some implications for protein unfolding. J. Phys. Chem. 86, 3870-3881 (1982). DOI: 10.1021/j100216a033

14. Rajagopal, K. \& Jayabalakrishnan, S. S. Effect of Temperature on Volumetric and Viscometric Properties of Homologous Amino Acids in Aqueous Solutions of Metformin Hydrochloride. Chin. J. Chem. Eng. 18, 425-445 (2010). DOI: 10.1016/S1004-9541(10)60241-8

15. Banipal, T. S., Kaur, J. \& Banipal, P. K. Interactions of some amino acids with aqueous manganese chloride tetrahydrate at $\mathrm{T}=(288.15$ to 318.15$) \mathrm{K}$ : A volumetric and viscometric approach.J. Chem. Thermodyn. 48, 181-189 (2012). DOI: 10.1016/j.jct.2011.12.012

16. Yan, Z., Wang, J., Liu, W. \& Lu, J. Apparent molar volumes and viscosity B-coefficients of some $\alpha$-amino acids in aqueous solutions from 278.15 to $308.15 \mathrm{~K}$. Thermochim. Acta 334, 17-27 (1999). DOI: 10.1016/ S0040-6031(99)00107-0

17. Kell, G. S. Density, thermal expansivity, and compressibility of liquid water from 0.deg. to 150.deg.. Correlations and tables for atmospheric pressure and saturation reviewed and expressed on 1968 temperature scale. J. Chem. Eng. Data 20, 97-105 (1975). DOI: 10.1021/je60064a005

18. Riddick, J. \& Bunger, W. Organic Solvents (Techniques of chemistry). II, (Wiley-Interscience, 1970).

19. Yang, J.-Z., Lu, X.-M., Gui, J.-S., Xu, W.-G. \& Li, H.-W. Volumetric properties of room temperature ionic liquid 2: The concentrated aqueous solutions of \{1-methyl-3ethylimidazolium ethyl sulfate + water $\}$ in a temperature range of $278.2 \mathrm{~K}$ to $338.2 \mathrm{~K}$. J. Chem. Thermodyn. 37, 1250-1255 (2005). DOI: 10.1016/j.jct.2005.03.002

20. F.R.S, S. D. O. M. K. B. E. XXVIII. Solute molecular volumes in relation to solvation and ionization. Lond. Edinb. Dublin Philos. Mag. J. Sci. 8, 218-235 (1929). DOI: $10.1080 / 14786440808564880$

21. Franks, F. \& Smith, H. T. Apparent molal volumes and expansibilities of electrolytes in dilute aqueous solution. Trans. Faraday Soc. 63, 2586-2598(1967). DOI: 10.1039/
TF9676302586

22. Shekaari, H. \& Jebali, F. Densities and electrical conductances of amino acids + ionic liquid ([HMIm] Br) $+\mathrm{H} 2 \mathrm{O}$ mixtures at 298.15 K. Fluid Phase Equilibria 295, 68-75 (2010). DOI: 10.1016/j.fluid.2010.04.002

23. Samanta, T. \& Saharay, S. K. Volumetric and viscometric studies of glucose in binary aqueous solutions of urea at different temperatures. J. Chem. Thermodyn. 42, 1131-1135 (2010). DOI: 10.1016/j.jct.2010.04.012

24. Hepler, L. G. Thermal expansion and structure in water and aqueous solutions. Can. J. Chem. 47, 4613-4617 (1969). DOI: $10.1139 / \mathrm{v} 69-762$

25. Frank, H. S. \& Evans, M. W. Free Volume and Entropy in Condensed Systems III. Entropy in Binary Liquid Mixtures; Partial Molal Entropy in Dilute Solutions; Structure and Thermodynamics in Aqueous Electrolytes. J. Chem. Phys. 13, 507-532 (1945). DOI: 10.1063/1.1723985

26. Ali, A. \& Shahjahan. Volumetric, viscometric and refractive index behavior of some $\alpha$-amino acids in aqueous tetrapropylammonium bromide at different temperatures. J. Iran. Chem. Soc. 3, 340-350 (2006). DOI: 10.1007/ BF03245957

27. Pal,A. \& Chauhan, N. Volumetric behaviour of amino acids and their group contributions in aqueous lactose solutions at different temperatures. J. Chem. Thermodyn. 43, 140-146 (2011). DOI: $10.1016 /$ j.jct.2010.08.004

28. Belibaĝli, K.B.\& Ayranci, E. Viscosities and apparent molar volumes of some amino acids in water and in $6 \mathrm{M}$ guanidine hydrochloride at $25^{\circ}$ C. J. Solut. Chem. 19, 867-882 (1990). DOI: $10.1007 / \mathrm{BF} 00653072$

29. Liu, C., Zhou, L. \& Lin, R. Volumetric Properties of Amino Acids in Aqueous N-methylacetamide Solutions at 298.15 K. J. Solut. Chem. 39, 1253-1263 (2010). DOI: 10.1007/ s10953-010-9585-y

30. Singh, M., Pandey, M., Yadav, R. K. \& Verma, H. S. Thermodynamic studies of molar volume, pair and triplet interactions at increasing side-chain length of $\alpha$-amino acids in aqueous potassium chloride solutions at different concentration and 310.15 K. J. Mol. Liq. 135, 188-191 (2007). DOI: 10.1016/j.molliq.2006.12.029

31. Shahidi, F., Farrell, P. G. \& Edward, J. T. Partial molar volumes of organic compounds in water. III. Carbohydrates. J. Solut. Chem. 5, 807-816 (1976). DOI: 10.1007/ BF01167236

32. Millero, F. J., Lo Surdo, A. \& Shin, C. The apparent molal volumes and adiabatic compressibilities of aqueous amino acids at 25.degree.C. J. Phys. Chem. 82, 784-792 (1978). DOI: $10.1021 / \mathrm{j} 100496 \mathrm{a} 007$

33. Wang, X., Li, G., Guo, Y., Zheng, Q., Fang, W., Bian, P. \& Zhang, L. Interactions of amino acids with aqueous solutions of hydroxypropyl- $\beta$-cyclodextrin at different temperatures: A volumetric and viscometric approach. $J$. Chem. Thermodyn. 78, 128-133 (2014). DOI: 10.1016/j. jct.2014.06.016 
34. Pal, A. \& Kumar, S. Volumetric and ultrasonic studies of some amino acids in binary aqueous solutions of $\mathrm{MgCl} 2 \cdot 6 \mathrm{H} 2 \mathrm{O}$ at $298.15 \mathrm{~K}$. J. Mol. Liq. 121, 148-155 (2005). DOI: 10.1016/j.molliq.2004.12.003

35. Páez, M. S., Alvis, A. \& Arrazola, G. Propiedades Volumétricas de Trifluorometanosulfonato de 1-etil-3metilimidazolio en Solución acuosa de Tiosulfato de Sodio Pentahidratado a Diferentes Temperaturas. Inf. Tecnológica 26, 105-112 (2015). DOI: 10.4067/S071807642015000500014

36. Páez, F. A., Páez, M. S. \& Lamadrid, A. P. Interactions of glycine in aqueous solutions of 1-butyl-3methylimidazolium tetrafluoroborate at different temperatures. Quím. Nova 37, 418-425 (2014). DOI: 10.5935/0100-4042.20140078

37. Páez, M. S., Vergara, M. K. \& Pérez, O. A. Propiedades Volumétricas de la DL-Alanina en Soluciones Acuosas del Líquido Iónico Cloruro de 1 -Butil-3-Metilimidazolio a las Temperaturas desde 283.15 hasta $313.15 \mathrm{~K}$. Inf. Tecnológica 26, 113-120 (2015). DOI: 10.4067/S0718-
07642015000500015

38. Tomé, L., Domínguez, M., Claudio, A., Freire, M., Marrucho, I., Cabeza, O.\& Coutinho, J. On the Interactions between Amino Acids and Ionic Liquids in Aqueous Media. J. Phys. Chem. B 113, 13971-13979(2009). DOI: 10.1021/ jp906481m

39. Banerjee, T. \& Kishore, N. Interactions of Some Amino Acids with Aqueous Tetraethylammonium Bromide at 298.15 K: A Volumetric Approach. J. Solut. Chem. 34, 137-153 (2005). DOI: 10.1007/s10953-005-2746-8

40. Singh, S. K., Kundu, A. \& Kishore, N. Interactions of some amino acids and glycine peptides with aqueous sodium dodecyl sulfate and cetyltrimethylammonium bromide at $\mathrm{T}=298.15 \mathrm{~K}$ : a volumetric approach. J. Chem. Thermodyn. 36, 7-16 (2004). DOI: 10.1016/j.jct.2003.09.010

41. Banipal, T. S., Kaur, J., Banipal, P. K. \& Singh, K. Study of Interactions between Amino Acids and Zinc Chloride in Aqueous Solutions through Volumetric Measurements at T $=(288.15$ to 318.15$)$ K. J. Chem. Eng. Data 53, 1803-1816 (2008). DOI: $10.1021 /$ je8001464 